\title{
A new brace for the treatment of scoliosis
}

\author{
M. G. Antonelli ${ }^{1}$, P. Beomonte Zobel ${ }^{1}$, P. Raimondi ${ }^{1}$, \\ T. Raparelli ${ }^{2} \&$ G. Costanzo ${ }^{3}$ \\ ${ }^{I}$ Department of Mechanical Engineering, Energy and Management, \\ University of L'Aquila, Italy \\ ${ }^{2}$ Department of Mechanics, Turin Polytechnic, Italy \\ ${ }^{3}$ Department of Orthopaedic Surgery, \\ University of Rome "La Sapienza" polo pontino, Italy
}

\begin{abstract}
In this paper a new brace for the treatment of scoliosis is proposed. It uses pressurized air, by many air pockets, to apply the corrective thrusts to the rib cage and it can apply the thrusts at many levels of the spine. Moreover, the thrusts can be modified and monitored. In this way the corrective action on the spine can be more effective. The design of the prototype of the new brace is presented together with the design, the prototyping and the validation of the air pocket. Finally the first experimental tests on a simplified prototype of the brace are presented.

Keywords: scoliosis brace, spinal orthoses, brace design, pneumatic pad.
\end{abstract}

\section{Introduction}

Scoliosis is a complex structural deformity of the spine in which there is an abnormal curvature of the vertebral column with respect to the 3 spatial axes. It appears as a lateral curvature on the frontal plane, a modification of the curves on the sagittal plane and a vertebral rotation on the horizontal plane [1]. Idiopathic scoliosis is the most common type and its origin is unknown. Brace treatment for idiopathic scoliosis has good support in published studies [1,2].

The brace is a tentative to slow the progression of the curve. Several different braces are used in the treatment of scoliosis, and most of them work on the curve via the pressure they exert on the rib cage, usually on 3 points, "to push against" the progressive abnormal curvature of the spine. The choice of the brace depends on the age, on the spine condition, but overall the doctor will select one based on 
his experience with the different orthoses. A few centers treat young children, with severe idiophatic scoliosis, with a body cast that is fixed on the body and can be removed only permanently (it is periodically substituted because of the growth of the child and of the evolution of the scoliosis). Very common for the treatment of scoliosis are the removable braces, and many different types are on the market. The most used are Milwaukee and Boston, designed in the USA, Cheneau and Lyon, designed in France, and La Padula, designed in Italy.

In this paper a new brace for the treatment of scoliosis is proposed. It uses pressurized air, by many air pockets, to apply the corrective thrusts to the rib cage and it can apply the thrusts at many levels of the spine. Moreover, the corrective pressure inside the pockets can be modified and monitored. In this way the action on the spine can be more effective. The design of the prototype of the new brace is presented together with the design, the prototyping and the validation of the air pocket. Finally, the first experimental tests on a simplified prototype of the brace are presented.

\section{The use of brace in the treatment of scoliosis}

Since 400 b.c Ippocrate tried to reduce the scoliosis curvature by a table, to which the patient was tied, and a person that hopped on him pushing the spine. In the following centuries many scientists (Galeno, Parrè, Delpech and others) were engaged in finding a therapeutic method for scoliosis: the application of mechanical pushing to convex parts of the spine, the extension of the spine by a traction force or by the weight of the patient. But only in the $1840 \mathrm{~s}$ did the activity of orthopaedic medical centers in France specifically devoted to the correction of the scoliosis start. In the following decades the scientists move from the traction fixed devices to different ideas of brace. The brace of ShanzMilwaukee (1945) and the optimised version of Blount and Schmidt (1958) are the most significant ones of that period. The final part of the XX century saw a growing interest in research activities on scoliosis and on the braces. Many different braces were proposed in this period, often "specialised" for a specific curvature, also considering the age of the patient: the Lyon brace, the Boston brace, the Cheneau brace, the Lapadula brace, etc.

The use of removable braces is recommended in scoliosis curvatures with the Cobb angle between $20 \pm 5^{\circ}$ and $40 \pm 5^{\circ}$ [1], depending on the authors. In this case the therapy is most effective with physical rehabilitation. Therapeutic rehabilitation is addressed for Cobb angle less than $15 \pm 5^{\circ}$, while the fixed brace and the surgical treatment is the therapy for the Cobb angle greater than $40 \pm 5^{\circ}$ [1]. The prognosis of scoliosis is based on some clinical data (hump, Cobb angle, torsional angle, age, etc.) and on its localization and curve pattern. After this step, the clinician specializing in spinal diseases will choose the specific brace, if necessary and useful. The brace works via the thrust it exerts on the rib cage to push against the abnormal curvature of the spine and to reduce the local spasticity. The brace applies thrusts directly onto the rib cage, primary correction effect; consequently, some reaction thrusts arise at the contact area between the brace and thorax. 
a)

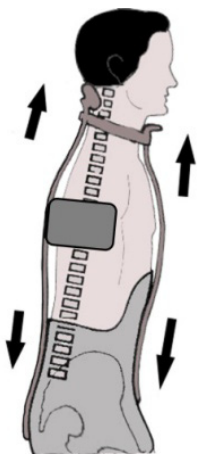

b)

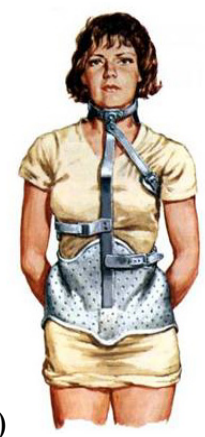

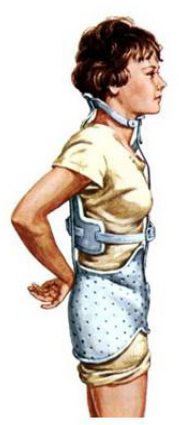

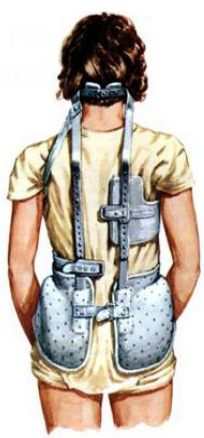

Figure 1: a) Sketch to show how a Milwaukee brace works [6], b) Milwaukee brace [7].

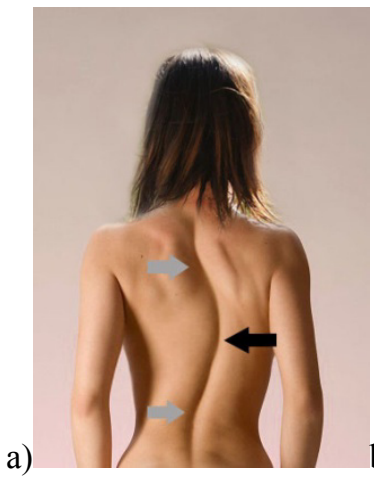

b)

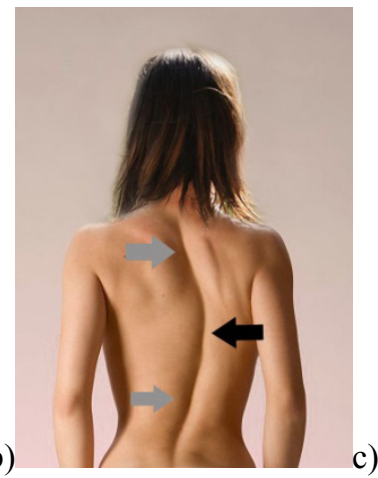

c)

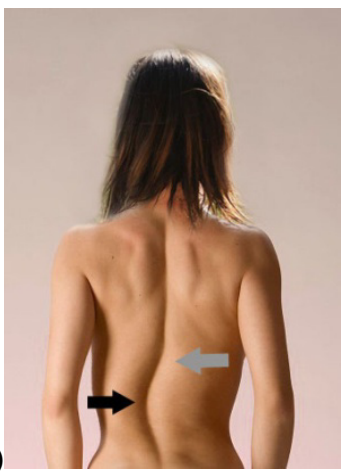

Figure 2: Some curvatures of the scoliotic spine: a) thoracic, b) thoracolumbar, c) lumbar.

Removable brace treatment for idiopathic scoliosis is a fundamental therapy, but the brace has to be light, patient compliant, constructed by an orthotist specializing in the construction of the prescribed brace system. Many brace systems have been proposed, as previously described. Each brace has its own characteristic for a specific correction of the spine. For example the Lyon brace is indicated for lumbar and for thoracolumbar curves. The Cheneau brace has three different versions: for single lumbar curves, for lumbar curves and for thoracic curve with an apex at L4/T10. The Milwaukee brace is the most common one for the treatment of scoliosis. It is used for thoracolumbar curves and for double curves. This brace uses two distinct principles: the 3 points thrust and the extension of the spine. The brace is linked to the pelvic region and has a top part to constrain the chin. The patient has to stay with the chin far from the constraint, and so a traction force is applied to the spine, fig. 1a). To obtain this traction force the Milwaukee brace has a pelvic shell modelled to reduce the lumbar lordosis. To understand how the brace works it is useful to see fig. 1b), 
where the typical parts of a Milwaukee brace are shown: the pelvic shell, the top part to constrain the chin, the lateral shell to apply the main thrust. In the figure 2, three different curvatures of the scoliotic spine are shown. In this figure are depicted the principal thrust, black arrow, and the reaction thrusts, the gray ones, that the brace applies to the spine. The reaction thrusts born in the pelvic shell and in the top part of the brace. The principal and the reaction thrusts do not succeed to apply the load to all the spine but just on the apex of the curvature and on the pelvic and on the neck regions. To increase the surface of the rib cage where the thrusts are applied, so that the correction pushing is applied to a greater part of the spine, some plates are used.

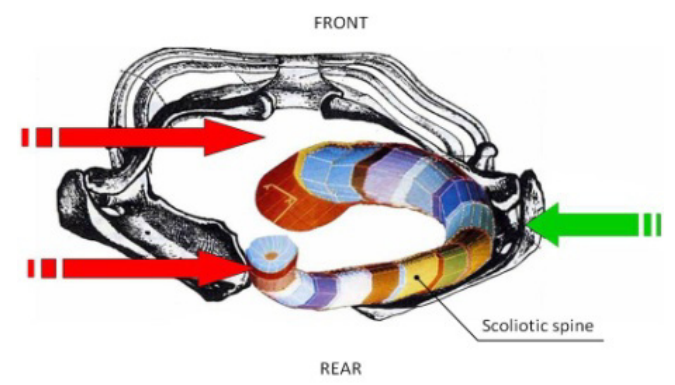

Figure 3: Scoliotic spine in a trasversal plane: on the right the main thrust and on the left the 2 reactions thrusts.

a)

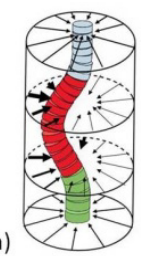

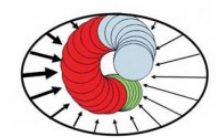

b)

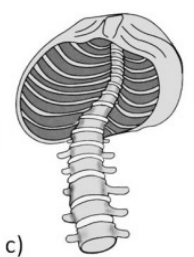

c)

Figure 4: a) Axonometric view of the scoliotic spine model, b) horizontal view of the s.s.m., c) axonometric view of scoliotic spine and thorax.

\section{The new brace}

It is important to consider that the spine, and obviously the scoliotic one too, is formed by vertebrae and each of them has 6 degrees of freedom (dof) with respect to the adjacent one. These 6 dof have a limited movement range, but there exists the possibility of a collapse of the spine, where it has the maximum value of the instability, when the patient wears a brace with 3 thrusts, fig. 3 . The elicoidal shape of the scoliotic spine needs more thrusts relating to the extension of the curvature, figs. 4a), 4b). In fact, the scoliotic curvature extended for 10 vertebrae requires more thrusts than a curvature extended for only 5 vertebrae. From our point of view the spine can be considered as made by 
24 cylindrical bodies, the vertebrae; the ends of each body articulate by pads of elastic or cartilaginous tissue with those of adjacent ones. This cylinder of 24 segments with a specific configuration, depending on the shape of the vertebrae, is linked to the rear part of the thorax, from the internal side, with a rotational joint, so that the spine can swing relative to the thorax, fig. 4c). The braces use the thrusts on the rib cage to obtain the displacement, i.e. traslation and rotation, of the spine for correcting it. Greater is the surface where the thrust is applied lesser is the possibility that the spine moves in unwanted directions.

\subsection{The design of the brace}

The brace proposed is based on the "global thrust" on the entire thorax. The idea is to have thrusts at many levels of the spine, not only at 3 levels as with a traditional brace. To obtain this behaviour the structural part of the brace is made with plastic shell, and in the internal part of the shell a covering of air pockets is fixed to it. The covering is made by rubber air pockets, in contact with the thorax, that are pressurized individually to obtain the required value of thrust. Moreover the corrective pressure inside the pocket can be modified and monitored.

The use of the brace is as follows. At first the value of thrusts to be applied to the rib cage for reduction of the scoliotic curvature has to settle. Then the value of pressure of the air pockets to obtain those thrusts has to be calculated for each point of the scoliotic curvature. Finally each air pocket can be inflated at the specific pressure value. In this way many thrusts are applied to the scoliotic curvature of the spine, one for each vertebra and composed by more radial components, except for the expanding part of the thorax.

\subsection{The design and the prototype of the air pocket}

The knowledge of values of the thrust in a scoliosis brace is necessary to design the air pocket. For this goal some experimental tests have been scheduled with 4 scoliotic patients, that were selected from the clinician, to measure the contact pressure, i.e. the thrust, between the pads and the rib cage. The utility of knowing the thrust value at the pad interface is due to the conception of the new brace. The air pockets are very similar to the pads, with the difference that they can be distributed all around the rib cage. All these patients have the same characteristics: a diagnosis of scoliosis and a therapy with a 3-point Cheneau brace. Other personal information was registered in a database but was not used to select them, because the goal of these tests was only to acquire typical values of thrust applied by the pads of a scoliosis brace. The measurement set-up was made by a sensors' matrix, a conditioning unit, all manufactured by Medizintechnik Gmbh, a data acquisition board NIDAQ 6036E of National Instruments and a Personal Computer. The sensors' matrix has a dimension of 80 x $85 \mathrm{~mm}$ and a thickness of $2.3 \mathrm{~mm}$. It is made by 8 couples of pressure and temperature sensors that are positioned in two rows and each couple of pressure and temperature sensors are annealed in a gel cell to distribute the pressure on the sensor and to protect against the shock. Each couple of sensors appears as a 
single sensor and it has a dimension of $11,5 \times 27,5 \mathrm{~mm}$. The maximum value of the pressure that each sensor can measure is 2 bars with a maximum error of $5 \%$ (i.e. \pm 50 mbar). The figure 5 shows a photo of the matrix of sensors and a draw with the disposition of the 8 sensors.

A simple protocol was defined to conduct this test. The protocol is as follows:

1. the clinician gives the patient complete information about the experimental test (goal, procedure, risk) and the patient accepts by signing a consensus agreement;

2. the patient wears the brace in the correct way;

3. the sensor is inserted in a disposable sterile small bag;

4. a data acquisition starts to test the sensor and to read the offset, i.e. the zero position of the meter, before inserting the sensor;

5. the brace is removed from tension to insert the sensor between the thrust point and the rib cage. The positioning of the sensor is a delicate step because it is necessary to avoid the presence of creases and the sensor has to remain finely in contact with the thorax and the pad;

6. the brace is tensioned and the first data acquisition starts with the patient in erect position; a check on the acquired data is made, so that if something is wrong the test can be repeated;

7. a second acquisition starts with the patient in seated position; a check on the acquired data is made, so that if something is wrong the test can be repeated;

8. is it the first measurement point? If the answer is yes, go back to the point 5 . to go on with the second measurement point. If not, removes the brace from tension to recover the sensor: the test with the patient is completed.
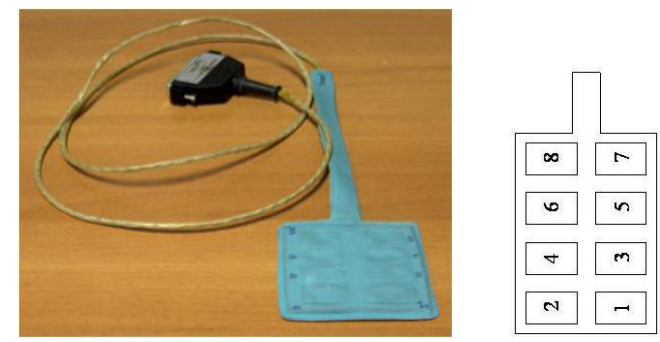

Figure 5: The 8 sensors' matrix and the position of these sensors.

A grid of the back was used to locate the position of the measurements points for each patient, fig. 6a). The measurement's points of the patients, from 1 to 4 , were respectively: $2 \mathrm{C}$ and $4 \mathrm{~B}, 2 \mathrm{C}$ and $3 \mathrm{~B}, 3 \mathrm{C}$ and $4 \mathrm{~A}, 1 \mathrm{~B}$ and $1 \mathrm{C}$. An example of acquired data from the test is reported in fig. 6b)', 6b)" (sample frequency of $1 \mathrm{kHz}$ and about 10.000 samples acquired), where it is possible to see the acquired pressure raw data for each of the 8 sensors, that measure the pressure between pad and rib-cage, and the total force $\mathrm{F}$ of the sensors matrix calculated with this formula:

$$
F=A \cdot \sum_{1}^{8} p_{i} .
$$


where $\mathrm{p}_{\mathrm{i}}$ is the pressure measured from the sensor $\mathrm{i}$ and $\mathrm{A}$ is the area of each sensor $\left(316 \mathrm{~mm}^{2}=11,5 \times 27,5\right)$. The data are oscillating because of the breathing, i.e. the change of the volume of the rib cage modifies the contact pressure and the thrusts. The maximum value of thrust that was measured is $37 \mathrm{~N}$ (patient n. 1 in seated position, point $2 \mathrm{C}$ ). In all the tests the maximum pressure was measured in seated position. This is due to the kyphotic position of the upper back in many people when seated. Some measurements have shown very low level of pressure, less than 4 N. Probably, in these cases at the interface pad rib cage a contact-no contact situation occurs.

a)

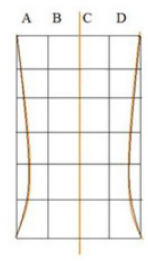

b')

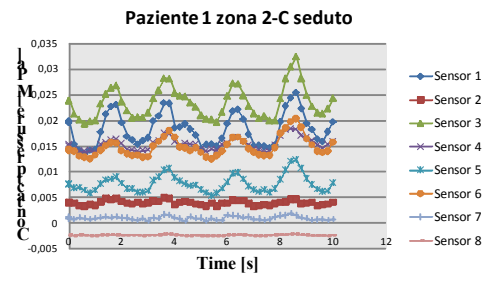

b")

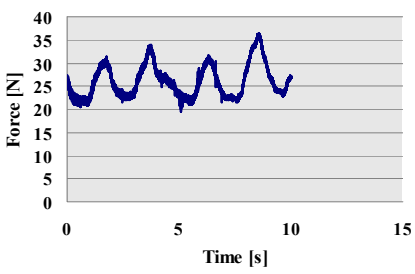

Figure 6: a) Grid of the back to locate the measurement points, b') example of the output of the 8 sensors' matrix and, b") total thrust applied.

After this fundamental preliminary step, the technical issues of the thrust element can be defined as:

- maximum level of force, on a area of about $80 \times 80 \mathrm{~mm}^{2}$, not less than $40 \mathrm{~N}$;

○ possibility to modify the thrust force during the brace therapy;

○ pneumatic technology to fix the value of pressure, i.e. the thrust, at the desired value;

○ architecture of many thrust elements, integrated each other, so that a covering of air pockets inside the shell is possible, to apply the thrusts where they need;

- soft contact with the thorax, for maximum comfort of the brace.

The designed thrust element is an air pocket with a squared shape. The air pocket is made of two layers of elastomeric material. To obtain a chamber that can be pressurised, the two layers are linked each other on the edge and a hole is used to inlet and to outlet the air from the pocket by a leak free valve. This valve is also used as fixing device to the shell. Pressurizing the air inside the pocket the volume grows as a balloon, so that a thrust is obtained "contrasting" the expansion of this volume. The silicone rubber was used as material, for its softness and its compatibility with the skin, and the manufacturing process was defined and tested by a technological set up. Because of the silicone behaviour 
the output force depends on both the pressure inside the pocket and its volume. In fact the elastomeric materials have a non-linear $\sigma-\varepsilon$ relationship. For this reason it was useful to define a numerical model to obtain a useful design tool. In this way the relationship among the main design parameters of the air pocket with the goal of optimising the driver design can be studied. The numerical model was defined using the Finite Element Technique by the ANSYS code [3]. Other details can be found in [4]. To validate the model a silicone prototype was constructed by the same technology that was used to make the air pockets for the brace, fig. $7 \mathrm{a}$.

c')$$
\text { a) }
$$
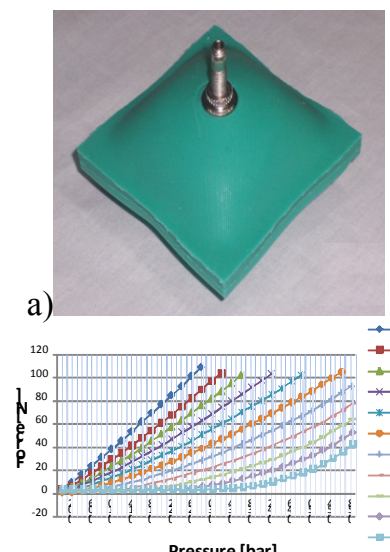

Pressure [bar] b)
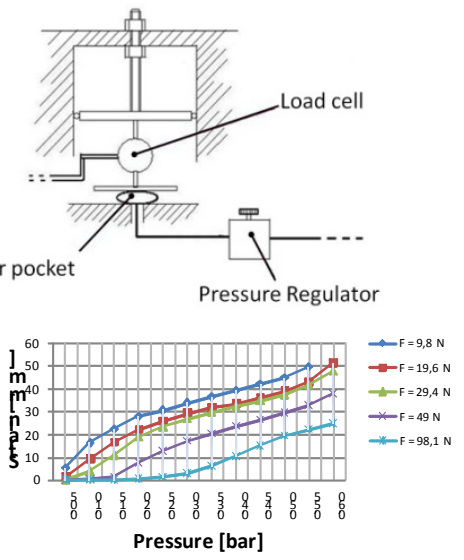

Figure 7: a) Prototype of the air pocket, b) set up for the isometric tests, c') results obtained in isometric and c'") isotonic tests.

The design of the air pocket and the tests on the prototype gave the following results: thickness: $8 \mathrm{~mm}$; square dimension: 100x100 mm; maximum force: 100 $\mathrm{N}$; maximum test pressure: 0.60 bar; burst pressure, at null deformation: 10 bar. Some problem arose in doing the burst test, because of the valve that is fixed to the wall by a nut. When the volume of the driver grows the fixing hole of the valve become larger. For this reason with free deformation of the air pocket the valve is ejected at a pressure of about 0.55 bar. In the application inside the brace the maximum deformation at design level is less than $10 \mathrm{~mm}$. At this value the burst pressure is more than 4 bar, so that it is correct to settle the maximum operative pressure at 0.65 bar.

Other experimental tests on the prototypes of air pocket were made in the two characteristic conditions: isometric and isotonic. The design and construction of a simple test bed was necessary to perform these tests. The isometric test consists in constant deformation experiments. The air pocket keeps its deformation by constraints and the force versus pressure inside the chamber is computed. The constraints are made by two simple aluminium plates. The deformation is fixed at different values by a simple screw-nut system that links the two plates. The deformation is measured by a measuring rod and a load cell on the screw is used 
for the traction force. The pneumatic components are a pressure regulator, connected to the pneumatic line, and a flexible tubing for the connection with the pocket. A manometer measures the air pressure and a portable tension-meter gives the tension value of the load cell that is used to calculate the traction force. The figure $7 \mathrm{~b}$ shows the experimental set up for the isometric tests of the device.

The isotonic test consists in constant force experiments. The device is free to extend and the displacement versus pressure are computed. The test bed is similar to the previous one. The aluminium plates are not linked each other. The constant load is obtained by gravitational mass located on the aluminium plate. The isotonic tests were performed in this manner. On the upper aluminium plate a mass is loaded and during the test the pressure $\mathrm{p}$ versus the displacement Dh of this plate is recorded. The test starts with no pressure inside the chamber and opening the pressure regulator in quasi-static manner the pressure reaches a maximum value of 0.6 bar. The isometric tests were performed by regulating the displacement $\mathrm{Dh}$ of the two aluminium plates with the screw-nut link from 0 to $40 \mathrm{~mm}$. The pressure inside the chambers is regulated in the same manner of the previous test, and recorded with the output of the load cell.

During both the tests, isotonic and isometric, the device shows an hysteretic behaviour, i.e. two distinct lines are obtained increasing the pressure inside the chambers from zero to a certain value and then decreasing to zero. This phenomenon is already described in literature for elastomeric materials [5], and the cause of the hysteresis seems to be the viscoelastic phenomenon. In this application it is neglected and the curves are constructed calculating the medium values.

\subsection{The construction of the brace and the first experimental tests}

The construction of the shell was obtained by a traditional CAD (Computer Aided Design) system normally used for the base frame of other brace, as Cheneau. The shell was modelled on the trunk of an healthy volunteer, inserting an offset, i.e. a backlash, of about $10 \mathrm{~mm}$ on the upper part. This offset was necessary because of the thickness of the air pocket $(8 \mathrm{~mm})$. A grid of holes were performed on the rear side of the shell, with a correct arrangement to assembly the air pockets by valve and nut from the internal side. Two air pocket prototypes were used for this first experimental tests. Wearing the brace, made by the shell and the 2 air pockets one close to the other, the impression was of too large size, with the drivers in a contact-no contact condition. The measurement made by the sensors matrix confirmed this condition. For this reason a covering was necessary to have a uniform thickness in the internal side of the shell. This problem was due to the use of only 2 air pockets, but it will disappear with the construction of the complete prototype that needs something like 30 air pockets. A layer of material, similar to that used for the pad, with a thickness of about 5 $\mathrm{mm}$ was used. It was glued on the internal side of the shell, except where are assembled the 2 air pockets. After this modification the volunteer had good sensation wearing the brace, with a normal contact between thorax and brace, fig. 8a. The measurements confirmed this good behaviour of the brace, giving values of the thrust less than $0.5 \mathrm{~N}$. After this step the first experimental tests on 
the brace started to measure the thrusts due to a single air pocket. The test was carried out in two different position of the air pocket, approximately $3 \mathrm{~B}$ and $4 \mathrm{~B}$, increasing the pressure value from 0 to 0,65 bar. The measured thrusts, by the sensors matrix, in the two different positions of the pocket versus the air pressure are very similar, with a maximum value of $25 \mathrm{~N}$ at 0,65 bar. The graph that shows the thrust of one air pocket versus the air pressure is in the fig. $8 \mathrm{~b}$ ). The result shows that the maximum value of thrust does not meet the design value of $40 \mathrm{~N}$, but it is important that the shape of the graph is approximately linear. The deficit in the maximum value of the thrust that the driver can apply means that a rectangle of 4 air pockets, $200 \times 200 \mathrm{~mm}^{2}$, can apply a maximum thrust of $100 \mathrm{~N}$, instead of $160 \mathrm{~N}$. That's mean that this new brace could be really interesting considering that the complete brace has a covering of air pockets in the internal side.
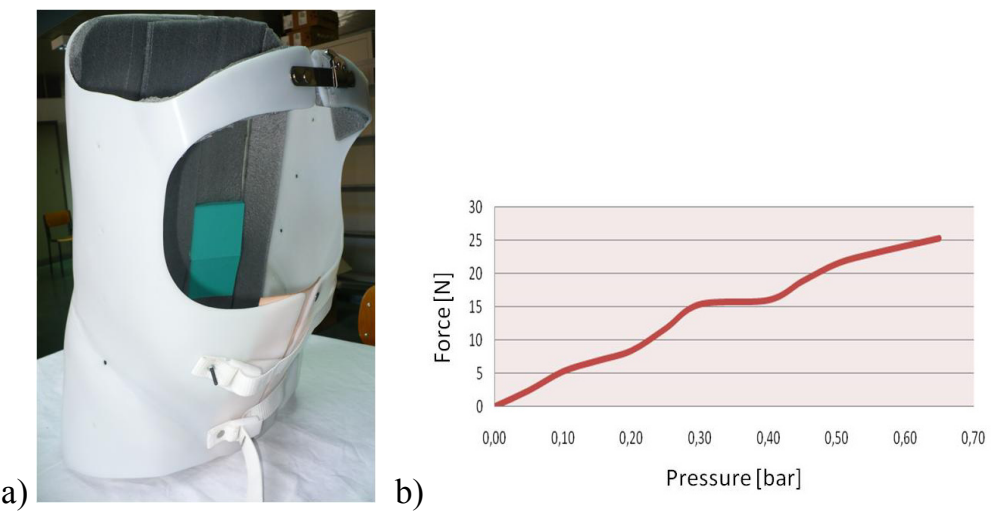

Figure 8: a) Prototype of the brace, b) total thrust applied by one air pocket.

\section{Conclusion}

In this paper a new brace for scoliosis is proposed. The basic idea of the new brace born to overcome the limitation of the 3 points thrust, typical of the most common removable braces used for scoliosis therapy. To obtain a better efficacy of the therapy, and to avoid the collapse of the spine, the thrusts should be applied at many levels of the spine. In this way the possibility that the spine moves in unwanted directions is avoided. Moreover the knowledge of the values of the thrusts is very useful to have quantitative data for the success of the brace therapy.

The structural part of the new brace is made with plastic shell and a covering of air pockets is fixed to the internal part. The pressure inside each air pocket can be regulated at the desired value. The paper shows the design, the prototyping and the experimental validation of the air pocket. Finally the first experimental tests on a healthy person with a simplified prototype of the brace with only 2 air pockets are shown. The results show that the idea of this new brace can be really interesting and the feasibility study here presented has given a positive answer. 
The work is in progress and the next step is a complete prototype of the new brace and, finally, a clinical validation.

\section{Acknowledgements}

The authors are thankful to Mr. Carlo Fricano and Mr. Alessandro Cipriani, as well as to Ortopedia Picena di Guiducci Quirino, Ascoli Piceno, Italy, for their kind support.

\section{References}

[1] Negrini S, Aulisa L, Ferraro C, Fraschini P, Masiero S, Simonazzi P, Tedeschi C, Venturin A., Italian guidelines on rehabilitation treatment of adolescents with scoliosis or other spinal deformities, Eura Medicophys $2005,41$.

[2] Labelle H, Dansereau J, Bellefleur C, Poitras B. Three-dimensional effect of the Boston brace on the thoracic spine and rib cage, Spine. 1996 Jan 1;21(1).

[3] ANSYS Theoretical manual, Swanson Analysis system Inc., Houston, USA, 1989

[4] Raparelli, T., Beomonte Zobel, P., Durante, F., Costanzo, G., Development of a pneumatic lumbar unloading device, IMG04 - International Conference on Intelligent Manipulation and Grasping, July 1-2, 2004, Genoa, Italy, pagg. 197-201

[5] Ferry J. D., Viscoelastic properties of polymers, John Wiley \& Sons, Inc., New York, 1980

[6] Viladot, R., Cohi, O., Clavel, S., Ortesi e protesi dell'apparato locomotore, (in italian) Ed.Verduci, 1988

[7] Simposi Clinici CIBA, (in italian), 1973 